\title{
THE OPTIMAL DESIGN OF FRACTIONAL SLIDING MODE CONTROL BASED ON MULTI-OBJECTIVE GENETIC ALGORITHMS FOR A TWO-LINK FLEXIBLE MANIPULATOR
}

\author{
Milad Pouya', Pooyan Vahidi Pashaki ${ }^{2}$
}

1 Department of Mechanical Engineering, University Campus 2, University of Guilan, Rasht, Iran, e-mail: mpouya@msc.guilan.ac.ir

2 School of Mechanical Engineering, Islamic Azad University, Science and Research Branch of Tehran (Central), Hesarak, Tehran, Iran, e-mail: pooyan.vahidi66@gmail.com

Received: 2017.05.15

Accepted: 2017.08.01

Published: 2017.09.03

\begin{abstract}
In this paper a novel optimal approach of control strategy is introduced by applying fractional calculus in the structure of sliding mode control for a range of dynamics system liable to ambiguity. So, a fractional sliding mode control was designed for dynamics of the two-link rigid-flexible manipulator. Furthermore, a multi-objective genetic algorithm was proposed in order to find the ideal variable structure of the sliding mode control. Optimal variables were achieved by the optimization of the conventional sliding mode control. Then the performance of both the conventional and the fractional sliding mode control were compared with respect to optimal variables. Results indicated that by applying the optimized fractional sliding mode control, the system's error was significantly reduced consequently tracking the desired value was done with a higher degree of accuracy and a smoother control action was achieved.
\end{abstract}

Keywords: fractional calculations, sliding mode control, two-link flexible manipulator, Pareto optimal, genetic algorithm.

\section{INTRODUCTION}

In recent years, a lot of researchers in engineering sciences have become interested in the application of non-integer order systems and as a result, concentrated on the element of fractional order systems. In the control theory, many studies have been conducted successfully on the design of the integer order (Zinober 1989, Das 2007, Aghababa 2015, Zhong et al. 2016). In recent years, through a better theoretical understanding of fractional calculations and the subsequent developments which are widely used in various fields of engineering sciences and by applying fractional order, operators in the controller structure, realised a new vision in the field of automatic control systems. Oldham and Spinner in (Zinober 1989) and in (Podlubny 1999) conducted research on the key components of fractional calculus, and fractional differential equations and Das (2007) focused on the engineering perspective of this issue. Aghababa (2016) introduced a fractional control method for chaos control of integer-order non-autonomous chaotic systems based on the sliding mode control. Zhong et al. (2015) developed fractional order sliding observer mode structures for the fractional order nonlinear system models. They investigated the asymptotic stability of error by Lyapunov stability analysis approach. Bisheban and Mahmoodabadi (2013) proposed the decoupled SMC technique to stabilize an inverted pendulum, which was optimized by Multi-objective particle swarm algorithm to reduce the normalized angle error of the pole and distance error of the cart, concurrently. 
Studies on fractional calculations are very widespread in the field of automatic control, and some of them are: Fractional systems in the context of feedback control (Zinober 1989), fractional PID controller (Hamamci 2007), and issues related to parameters selection using the Ziegler-Nichols method rules (Valério \& da Costa 2006). Das and Pan (2014) designed a fractional-order PID controller for an automatic voltage regulator system to measure objectives such as the set-point tracking, load disturbance, and noise rejection controller effort, in the Pareto optimal solution. Pan et al. (2015) proposed an active control policy design for a fractional order financial system, which considers multiple conflicting objectives as a nonlinear state feedback mechanism. Pan and Das (2015) designed a fractional-order PID controller for load-frequency control of two interconnected power systems. They developed multi-objective optimization frameworks based on the NSGAII and chaotic optimization to tune the gains and the fractional differ-integral orders of the PID controllers. Their results showed that the fractional PID controller system which optimized evolutionary algorithms could rapidly follow the desired output with higher precision and robustness.

In this paper, a fractional controller is proposed to eliminate chattering and tracks the same periodic two-link manipulator even with levels of high uncertainty. To deal with structural (lack of precision in the model) and non-structural uncertainties (lack of precision in order of system) in the control model, the fractional sliding mode controller was used. Fundamentally, SMC involves two parts: the plan of the surface in the state space so that the decreased order sliding movement fulfills the determination force of the architect; and the amalgamation of a control law and spasmodic sliding surface, such that the directions of the closed loop move- ment are coordinated towards the surface. For this purpose, a parameter selection method proposed using a multi-objective GA in order of sliding mode control of the variable structure system.

\section{FRACTIONAL CALCULATIONS}

Fractional analytics is a branch of mathematical science that investigates the possibility of getting real or complex number powers of the differentiation operator and generalizes the derivative or integral of a function to noninteger order, permitting calculations such as deriving a function to $1 / 2$ order (Panigrahi et al. 2013). Calculus gives a meaning to $d f / d t, d^{2} f / d t^{2}$ and $\int f(u) d u$ that they are respectively first and second order derivatives and first order integral. But what if the differentiation order is not an integer, according to these purposes, fractional calculations can be used. Fractional calculus was introduced in September $30^{\text {th }}, 1659$ in a letter L'Hopital wrote to Leibniz. After Leibniz (1695), other scientists including Euler (1730), Lagrange (1772), Laplace (1812), Abel (1822), Liouville (1832), Riemann (1876) and Grunwald (1838-1920) and Letnikoff (1837-1888) worked in the field. Fractional derivative with a basic definition as $\mathrm{D}_{\mathrm{t}}^{\alpha}$, which is a decision of differential and integral operators is characterized as follows:

$$
D_{t}^{\alpha}=\left\{\begin{array}{c}
\frac{d^{\alpha}}{d t^{\alpha}} \\
1 \\
\int_{a}^{t}(d t)^{-\alpha}
\end{array}\right.
$$

Where a and $\mathrm{t}$ are the limits of the operation and $\alpha \in \mathrm{R}$.

The Grunwald-Letnikov (GL) and the RiemannLiouville (RL) are two definitions used for the general fractional differ-integral. The GL is given as:

$$
{ }_{a} D_{t}^{\alpha} f(x)=\lim _{h \rightarrow 0}\left(1 / h^{q}\right) \sum_{m=0}^{\left[t-\frac{1}{h}\right]}(-1)^{j}\left(\begin{array}{l}
q \\
j
\end{array}\right) f(t-j h)
$$

$\mathrm{q}$ is the order of the derivatives and $\alpha$ is a constant related to the initial conditions. The RL definition is given as:

$$
{ }_{a} D_{t}^{q} f(t)=\frac{1}{\Gamma(n-q)} \frac{d^{n}}{d t^{n}} \int_{a}^{t} \frac{f(t)}{(t-\tau)^{q-n+1}} d \tau
$$

Fractional order differential equations are as steady as their integer orders partners, since systems with memory are usually more steady than their memory-less options (Baleanu \& Güvenç 2010).

By modifying the sliding mode control using a sliding surface containing fractional derivatives in order to design the sliding mode controller for two-link rigid-flexible manipulator. 


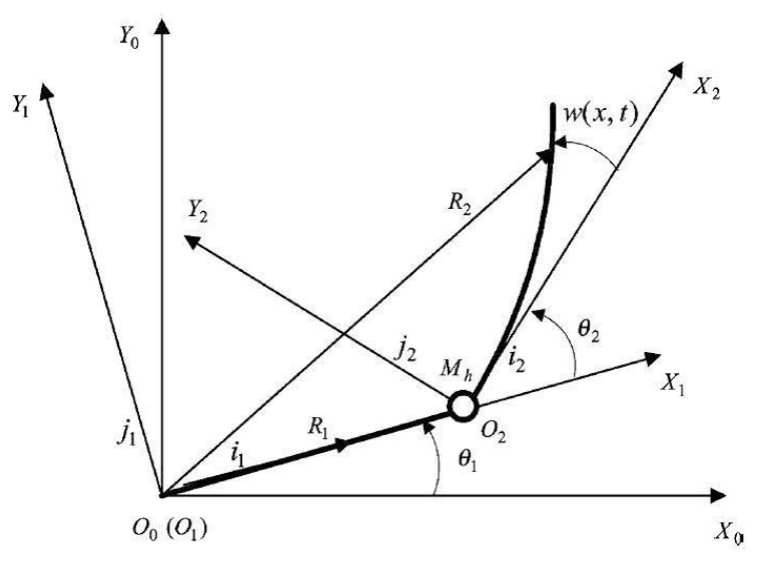

Fig. 1. The schematic diagram of a two-link rigid-flexible manipulator

\section{DYNAMIC EQUATIONS OF MOTION FOR THE TWO-LINK RIGID-FLEXIBLE MANIPULATOR}

The system considered in this study consists of two members which, as demonstrated in Fig. 1 , are connected to each other by a revolute joint and are only capable of planar motions. The first member is considered rigid, while the second is modeled as a flexible narrow beam. Longitudinal deformations are neglected in the second member. It is assumed that the second member can be bent freely in the horizontal plane, but can resist vertical bending as well as torsion. Hence, the Euler-Bernoulli theory may be used to describe the bending motions of the flexible member. In addition, the Lagrange equation can be used to derive the dynamic model of the twolink manipulator.

According to Fig. 1, $\mathrm{X}_{0} O Y_{0}$ is the fixed coordinate system, and $\mathrm{X}_{1} \mathrm{OY}_{1}$ and $\mathrm{X}_{2} \mathrm{OY}_{2}$ are the moving coordinate systems attached to the joints corresponding to the rigid and flexible links, respectively. In addition, $\theta_{1}$ and $\theta_{2}$ are the rotation angles of each of the links with respect to the $\mathrm{X}$ axis of their previous coordinate system, and $w(x, t)$ is the elastic transverse displacement of the flexible member. Since the bending motions of a beam do not impose significant axial vibrations, axial deformations were not included in our study. Two perpendicular pairs of unit vectors $\left(i_{p}, j_{p}\right)$ and $\left(i_{2}\right.$, $j_{2}$ ) attached to the moving coordinates of the links are shown in Fig. 1. The position vectors of the points on the Two-Link are $\mathrm{R}_{1}$ and $\mathrm{R}_{2}$, dynamic equations of motion are concluded. Selecting the $n$ first modes as the assumed-modes for the discretization procedure, the following centralized model is acquired for the system:

$$
M(X) \ddot{X}+K X+F(X, \dot{X}) \dot{X}=U
$$

Where $\mathrm{X}=\left[\theta_{1}, \theta_{2}, w_{1}, w_{2}, \ldots, w_{n}\right]^{T}$ is the vector of generalized coordinates, $x_{\theta}$ show the rigid body the $\mathrm{M}$ and $\mathrm{K}$ are the inertia and stiffness matrices, respectively, the vector $\mathrm{F}$ contains the nonlinear expressions associated with the Coriolis and centripetal forces, and $\mathrm{u}$ represents the inputs to the system (Pashaki et al. 2017; Pashaki \& Pouya 2016).

Dynamic equation of motion for manipulator can be written as:

$$
\left[\begin{array}{l}
u \\
0
\end{array}\right]=\left[\begin{array}{cc}
M_{\theta} & M_{\theta, w} \\
M_{w, \theta} & M_{w}
\end{array}\right]\left[\begin{array}{l}
\ddot{X}_{\theta} \\
\ddot{X}_{w}
\end{array}\right]+\left[\begin{array}{cc}
F_{\theta} & F_{\theta, w} \\
F_{w, \theta} & F_{w}
\end{array}\right]\left[\begin{array}{l}
\dot{X}_{\theta} \\
\dot{X}_{w}
\end{array}\right]+\left[\begin{array}{cc}
0 & 0 \\
0 & K_{w}
\end{array}\right]\left[\begin{array}{l}
X_{\theta} \\
X_{w}
\end{array}\right]
$$

Consider the reduced model which contains only the rigid part of the whole dynamic model of flexible robot arm:

$$
B u-F_{\theta} \dot{X}_{\theta}-K_{w} X_{\theta}=M_{\theta} \ddot{X}_{\theta}
$$

\section{SLIDING MODE CONTROL FOR TWO- LINK FLEXIBLE ROBOT ARM}

SMC was mentioned for the first time in 1950 by Emelyanov in the former Soviet Union (Emel'yanov 2007). It is a nonlinear control strategy that modifies the dynamics of a nonlinear system by utilization of a discontinuous control flag that powers the system to "slide" along a cross-segment of the system's typical behaviour. The various control structures are outlined so that directions dependably move towards a nearby locale with an alternate control structure. This part expresses the idea of sliding mode control for a Two-Link Flexible robot arm based on fractional order control. Initially, the sliding surface by using integer order of derivative (PD) is considered. Then fractional type of 
sliding surface is employed by using non-integer order of derivative $\left(P D^{\alpha}\right)$.

Now consider the following second-order nonlinear dynamic model which is described by (for convenience consider $X_{\theta}=x$ ) :

$$
\begin{aligned}
\dot{x}_{2 k-1}(t)=x_{2 k}(t) & \\
\dot{x}_{2 k}(t) & =\frac{b}{m} u-\frac{F_{\theta}}{m} \dot{x}-\frac{K_{w}}{m} x \quad k=1,2, \ldots, n(5) \\
x_{0}(t) & =x_{0}(t)
\end{aligned}
$$

Where, $x(t)$ is a state vector, $u(t) \in R$ is the control input. The initial desired state $x_{d}(0)$ must be $(0)=x\left(t_{0}\right), b_{k}(x), f_{k}(x)$ and $k=$ $1,2, \ldots, n$ operation and control of nonlinear dynamic system, respectively. The tracking error in the variable $\mathrm{x}$ is given as:

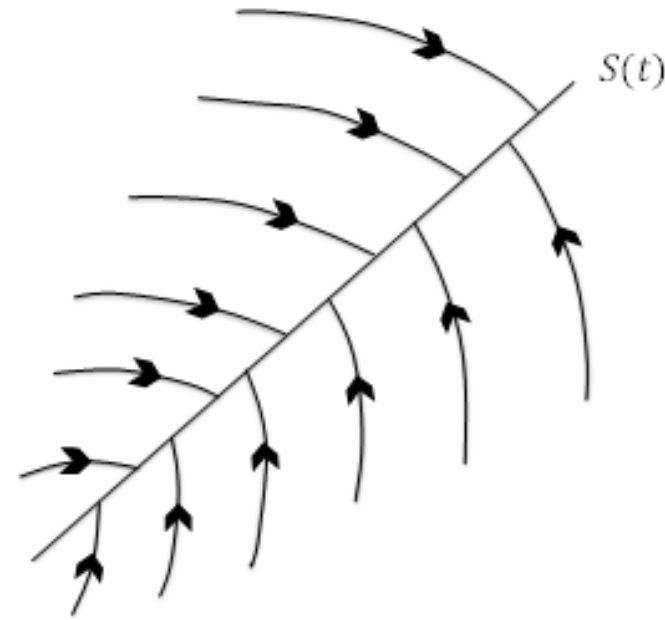

Fig. 2. Sliding condition

$$
x_{d}(t)=\left[x_{d}(t), x_{d}(t), x_{d}(t), \ldots x_{d}(t)\right]^{T} \quad \in R^{2 n}
$$

And the tracking error $\tilde{\mathrm{x}}(\mathrm{t}) \in \mathrm{R}^{2 \mathrm{n}}$ can be written as:

$\tilde{x}(t)=x(t)-x_{d}(t)$

The dynamics $\mathrm{f}(\mathrm{x}, \mathrm{t})$ is not exactly known, but estimated as $\hat{\mathrm{f}}(\mathrm{x}, \mathrm{t})$. The estimation error on $\mathrm{f}(\mathrm{x}, \mathrm{t})$ is assumed to be bounded by a known function $F$ :

$$
\left|f_{k}-\hat{f_{k}}\right| \leq \tilde{f_{k}}(x), \quad k=1,2, \ldots, n
$$

\section{CONVENTIONAL SLIDING SURFACE}

A conventional sliding surface can be defined as:

$$
s_{k}(\tilde{x}(t))=\lambda_{k} \tilde{x}_{2 k-1}(t)+\tilde{x}_{2 k} \quad \lambda_{k}>0
$$

Where, $\lambda_{k}$ is strongly a positive constant and the amount which determines the differential equation is stable. The issue of tracking of $x_{d}(t)=x(t)$ is equal to staying on the sliding surface for all $t>0$, in reality $\mathrm{S}=0$ speaks to a straight differential mathematical statement whose unique solution is 0 $=\tilde{x}(t)$, Thus, the issue of the tracking $\mathrm{x}_{\mathrm{d}}$ can be deduced to keeping the scalar amount of $\mathrm{s}$ at zero.

$$
s_{i} \dot{S}_{l} \leq-\eta_{i}\left|S_{i}\right|
$$

Where, $\eta$ is a strongly positive constant, $\mathrm{s}^{2}$ implies that the squared distance to the sliding surface reduced during all system trajectories. It diminished in all system directions. It contains directions that point towards the surface $s(t)$ and it includes trajectories towards the surface of $s(t)$. As shown in Figure 2, the system directions remained on the surface (Perruquetti 2002).

The derivative of (8) with respect to time can be represented by:

$$
\dot{S}_{k}(\tilde{x}(t))=\lambda_{k} e_{2 k}(t)+x_{d 2 k}(t)-\dot{x}_{d 2 k-1}(t)+\frac{b}{m} u-\frac{F_{\theta}}{m} \dot{X}_{\theta}-\frac{K_{W}}{m} X_{\theta}-\dot{x}_{d 2 k}(t) \quad \lambda_{k}>0
$$

Let $\mathrm{x}(\mathrm{t})$ changes and be the tracking error in the variable $\mathrm{x}_{\mathrm{d}}(\mathrm{t})$ :

$$
\begin{gathered}
\tilde{x}_{2 k}(t)=x_{2 k}(t)-x_{d 2 k}(t) \\
\tilde{x}_{2 k-1}(t)=x_{2 k-1}(t)-x_{d 2 k-1}(t)
\end{gathered}
$$

The derivative of (12) and (11) with respect to time can be represented by:

$$
\dot{\tilde{x}}_{2 k-1}(t)=\dot{x}_{2 k-1}(t)-\dot{x}_{d 2 k-1}(t)
$$




$$
\dot{\tilde{x}}_{2 k-1}(t)=\dot{\mathrm{x}}_{2 \mathrm{k}-1}(\mathrm{t})-\dot{x}_{d 2 k-1}(t)
$$

Then again substituting (11) into (5) yields:

$$
\begin{gathered}
\dot{e}_{2 k-1}(t)=e_{2 k}(t)+x_{d 2 k}(t)-\dot{x}_{d 2 k-1}(t) \\
\dot{\tilde{x}}_{2 k}(t)=\frac{b}{m} u-\frac{F_{\theta}}{m} \dot{X}_{\theta}-\frac{K_{w}}{m} X_{\theta}-\dot{x}_{d 2 k}(t) \quad k=1,2, \ldots, n
\end{gathered}
$$

Substituting (1) and (15) into (10) results:

$$
\dot{S}_{k}(\tilde{x}(t))=\lambda_{k}\left(e_{2 k}(t)+x_{d 2 k}(t)-\dot{x}_{d 2 k-1}(t)\right)+\frac{b}{m} u+\frac{F_{\theta}}{m} \dot{x}(t)-\frac{K_{W}}{m} x(t)-\dot{x}_{d 2 k}(t)
$$

In order to fulfil the sliding condition $\mathrm{s}=0$, despite uncertainty on the dynamics of, input control can be defined as:

$$
u_{k}(t)=\frac{m}{b}\left[\left(-\lambda_{k}\left(x_{2 k}(t)-\dot{x}_{d 2 k-1}(t)\right)-\frac{F_{\theta}}{m} \dot{x}(t)+\frac{K_{w}}{m} x(t)+\dot{x}_{d 2 k}(t)-k_{k} \operatorname{sgn}\left(S_{k}(t)\right)\right]\right.
$$

In this equation, the best approximation of an equivalent-control law that would achieve $\dot{\mathrm{s}}=0$ Also $k_{k}$ is the design is a switching feedback control gain and $k_{k} \geq \eta$.

During contact with the sliding surface due to the discontinuity in the sign function, chattering phenomenon was observed. In general, chattering must be omitted from the controller for proper execution. This can be achieved by replacing the sign function with the sat function and smoothing out the control irregularity in a thin limited layer with the thickness of $\Phi$ (Fig. 3). The switching surface that developed into the sat function will be depicted in next section (Mahmoodabadi et al. 2012).

$$
u_{k}(t)=\frac{m}{b}\left[\left(-\lambda_{k}\left(x_{2 k}(t)-\dot{x}_{d 2 k-1}(t)\right)-\frac{F_{\theta}}{m} \dot{x}(t)+\frac{K_{w}}{m} x(t)+\dot{x}_{d 2 k}(t)-k_{k} s a t\left(\frac{s_{k}(t)}{\Phi_{k}}\right)\right]\right.
$$

\section{Fractional sliding mode control for two link flexible manipulator}

Since the fractional-order sliding surface $P D^{\alpha}$ can be defined as follows:

$$
S_{k}(\tilde{x}(t))=\lambda_{k} \tilde{x}_{2 k-1}(t)+D^{\alpha} \tilde{x}_{2 k-1}
$$

Or it can be written as:

$$
S_{k}(\tilde{x}(t))=\lambda_{k} \tilde{x}_{2 k-1}(t)+D^{\alpha-1} \dot{\tilde{x}}_{2 k-1}
$$

And substituting (14) into (20) can be represented by:

$$
S_{k}(\tilde{x}(t))=\lambda_{k} \tilde{x}_{2 k-1}(t)+D^{\alpha-1}\left(\tilde{x}_{2 k}(t)+x_{2 d k}(t)-\dot{x}_{d 2 k-1}(t)\right)
$$

Taking derivative of (21) with respect to time can be represented by:

$$
\dot{S}_{k}(\tilde{x}(t))=\lambda_{k} \dot{\tilde{x}}_{2 k-1}(t)+D^{\alpha-1}\left(\frac{b}{m} u-\frac{F_{\theta}}{m} \dot{X}_{\theta}-\frac{K_{w}}{m} X_{\theta}-\ddot{x}_{d 2 k-1}(t)\right)
$$

Combination of (16) and (22), then by forcing $\dot{\mathrm{S}}_{k}=0$ :

$$
u_{k}(t)=\frac{m}{b}\left[D^{1-\alpha}\left(-\lambda_{k} \tilde{x}_{2 k-1}(t)\right)+\frac{F_{\theta}}{m} \dot{x}+\frac{K_{w}}{m} x+\ddot{x}_{d 2 k-1}(t)-k_{k} \operatorname{sgn}\left(S_{k}(t)\right)\right.
$$

By selecting $u_{k}(t)$ as equation (23), since the sign function in the meaning of the control law is a hard non-linearity, as we mentioned before, Function $\operatorname{sat}(s / \Phi)$ is substituted with $\operatorname{sgn}$ function to eliminate the chattering phenomenon in the sliding mode control, we then have:

$$
u_{k}(t)=\frac{m}{b}\left[D^{1-\alpha}\left(-\lambda_{k} \tilde{x}_{2 k-1}(t)\right)+\frac{F_{\theta}}{m} \dot{x}+\frac{K_{w}}{m} x+\ddot{x}_{d 2 k-1}(t)-k_{k} \operatorname{sat}\left(\frac{S_{k}(t)}{\Phi_{k}}\right)\right]
$$




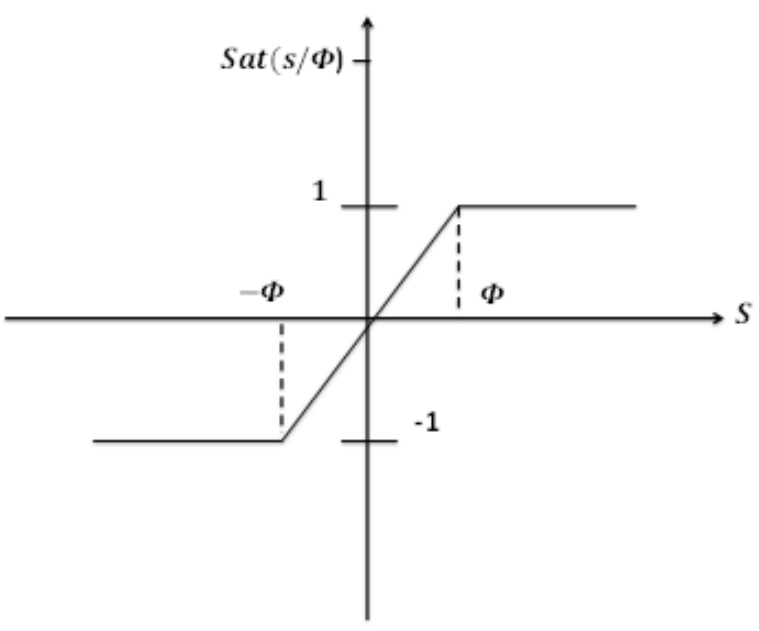

Fig. 3. Function $\operatorname{sat}(\mathrm{s} / \Phi)$ to eliminate the chattering phenomenon in the sliding mode control

\section{MULTI-OBJECTIVE OPTIMIZATION USING GENETIC ALGORITHM}

A large class of engineering problems include many optimization issues which help scientists to promote their results by using multi objective optimization.

Over the previous decade, various multi-objective algorithms have been recommended (Deb 2001; Fonseca \& Fleming 1993; Sbalzarini et al. 2000). An important explanation for this is their capacity to discover the Pareto set in one single recreation run (Deb et al. 2002). A sensible answer for a multi objective problem is to research a Pareto set in which each satisfies the objectives at an adequate level without being ruled by any other arrangement.

The goal of multi objective GA is to find as many of these solutions as possible. If reallocation of resources cannot improve one cost without raising another cost, then the solution is $\mathrm{Pa}$ reto optimal. A Pareto GA returns a population with many members on the Pareto front. The population is ordered based on dominance. A few unique algorithms have been suggested to be effectively related to different issues, for example (Sbalzarini et al. 2000).

Vector-Evaluated GA, Multi Objective GA, A Non-Dominated Sorting GA and Non-Dominated Sorting GA were used in the proposed research.

A multi objective optimization problem can be formulated as:

$$
\min \left(f_{1}(x), f_{2}(x), \ldots, f_{k}(x)\right)^{T}
$$

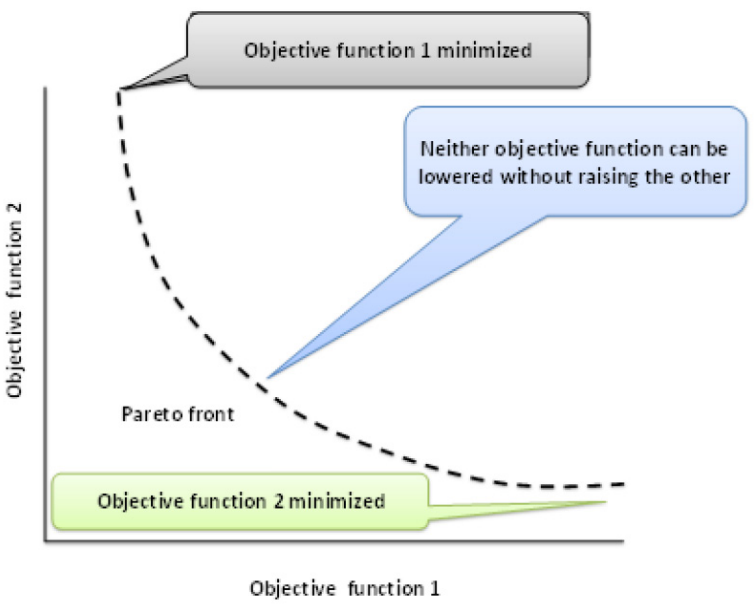

Fig. 4. Scheme of a multi-objective optimization

And also an n-dimensional decision vector is defined as:

$x=\left\{x_{1}, \ldots, x_{n}\right\}^{T}$

In the response space, $\mathrm{S}$ has been considered as $x \in \mathrm{S}$.

Where: $\left(\left(f_{1}(x), f_{2}(x), \ldots, f_{k}(x)\right)\right.$ are the k objectives functions, $\left\{\mathrm{x}_{1}, \ldots, \mathrm{x}_{\mathrm{n}}\right\}$ are $\mathrm{n}$ optimization parameters, and $\mathrm{S} \in \mathrm{R}$ is the solution of parameters space.

Pareto optimal definition: $\mathrm{x}^{*}$ is a Pareto optimal solution, MOG, if and only if there is no $\mathrm{x}$ (i.e., $\mathrm{x} \in \mathrm{S}$ ) that:

$$
f_{j}(x) \leq f_{j}\left(x^{*}\right) \quad j=1,2, \ldots, m
$$

$f_{j}(x) \leq f_{j}\left(x^{*}\right)$ for at least one objective function. The Pareto curve can include the trade-off point, which manages the balance between all objective functions. Figure 4 shows an optimal set related to a multi objectives optimization.

In view of the fact that the hitting time and the chattering issue are the most important factors that effectively influence the efficiency of the suggested controller, thin limited layer $\Phi_{k}$ that have immense effect on chattering phenomena and $K_{k}$ that will influence rate of synchronization considered as the variable optimization in multi-objective genetic algorithm. And in order to find the most suitable application of the fractional derivative.

In this multi-objective optimization, for conventional and fractional SMC optimization plan, two issues were considered:

- Error reduction in tracking the desired trajectory control system

- Reduction in control input (control effort). 
Where: $f_{1}(x)$ and $f_{2}(x)$ are the Objective functions and there are defined as the total amounts of tracking error and control input simultaneously in total simulation time, which can be written as follows :

$$
\left\{\begin{array}{l}
f_{1}(x)=\sum_{t}^{t_{n}}\left|e_{k}(t)\right| \\
f_{2}(x)=\sum_{t_{0}}^{t_{n}}\left|u_{k}(t)\right|
\end{array}\right.
$$

Objective functions simultaneously are effective in the performance of control system. It is desirable to have the fast reaching velocity to the switching hyper-plane in the hitting phase and slide to the origin with small chattering phenomena in the sliding phase. And also the Parameters of Multi-Objective Genetic Algorithm are defined as follows:

Population $=60 \quad$ crossover $=0.8$

Generations $=110 \quad k_{k}=[0,10]$

$\Phi_{k}=[0,2]$

\section{SIMULATION IMPLEMENTATION}

The optimal conventional and fractional sliding mode controller has been successfully employed by a planner to control the two-link flexible robot arm system (Pashaki \& Pouya 2017). Conventional sliding mode control PD Optimization was done with respect to optimization variables $\Phi$ and $k$ and Objective functions (26), and optimal parameters are chosen through as follow:

$$
\begin{aligned}
& \Phi=0.2421 \\
& K_{1}=3.3205, K_{2}=6.1032
\end{aligned}
$$

And others parameters are chosen as $\lambda_{1}=8$, $\lambda_{2}=8, \alpha=0.85$. Furthermore, the estimated value of the dynamic parameters of the manipulator given in Table 1. The simulation results based on $P D^{\alpha}$ sliding mode control and $P D$ Sliding Mode Control have been depicted in the Fig. 6 - 17 respectively. A fast tracking response is achieved by employing the $P D^{\alpha} \mathrm{SMC}$ in comparison with the response achieved by employing the $P D$ SMC. In addition, it can be seen that by employing the $P D^{\alpha} \mathrm{SMC}$ a smooth control action is achieved. The chattering of $u_{1}(t)$ and $u_{2}(t)$ are reduced in Figs. 16 and 17. From Figs. 12 and 13, it is observed that by employing the $P D^{\alpha} \mathrm{SMC}$, tracking performance are properly optimized and result in a faster tracking response with minimum reaching phase time in comparison with the $P D$ controller Figs. 6 and 7.

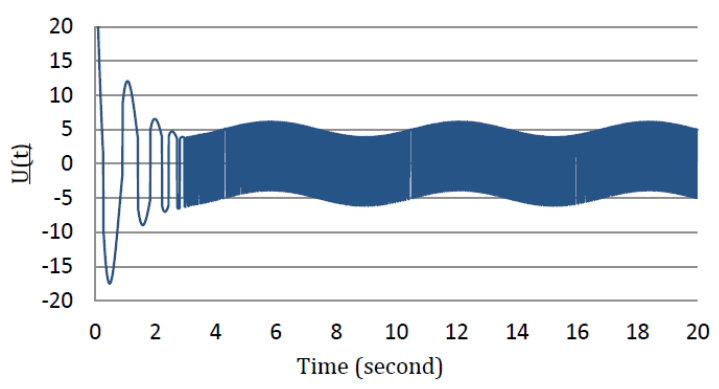

Fig. 5. PD Sliding Mode Control with sig function

Table 1. Manipulator properties

\begin{tabular}{|l|c|c|}
\hline \multicolumn{1}{|c|}{$\begin{array}{c}\text { Physical } \\
\text { parameters }\end{array}$} & Link1 & Link2 \\
\hline Length $(\mathrm{m})$ & $\mathrm{L}_{1}$ & $\mathrm{~L}_{2}$ \\
\hline Moment of inertia at the origin of the link $\left(\mathrm{kg} \cdot \mathrm{m}^{2}\right)$ & $\mathrm{j}_{1}=1.5 \cdot 10^{-3}$ & $\mathrm{j}_{2}=1.85 \cdot 10^{-4}$ \\
\hline Mass of the link $(\mathrm{kg})$ & 1.2 & 1.9 \\
\hline Mass at driving motor on the second link $(\mathrm{kg})$ & & \multicolumn{1}{|c|}{$\rho_{\mathrm{AL}}=7860$} \\
\hline Mass density of the second link & \multicolumn{2}{|c|}{$2.20 \cdot 10^{-10}$} \\
\hline Young modulus $\left(\mathrm{kg} / \mathrm{m}^{2}\right)$ & $\mathrm{E}_{1}=1.98 \cdot 10^{11}$ \\
\hline Second area moment of inertia $\left(\mathrm{m}^{4}\right)$ & & $\mathrm{E}_{2}=1.98 \cdot 10^{11}$ \\
\hline
\end{tabular}

Table 2. Result of the controller performances

\begin{tabular}{|l|c|c|}
\hline \multicolumn{1}{|c|}{ Controller } & PD & PD $^{\alpha}$ \\
\hline Reaching time 1 (s) & 1.83 & 0.48 \\
\hline Reaching time 2 (s) & 1.99 & 0.45 \\
\hline The absolute total error1 & 93.3289 & 10.0561 \\
\hline The absolute total error2 & 182.4476 & 9.5760 \\
\hline
\end{tabular}




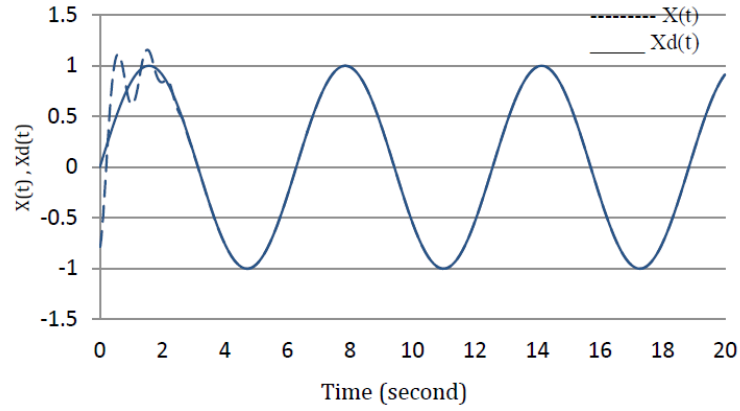

Fig. 6. Tracking response of $P D$ sliding mode control based on Multi objective Genetic Algorithm (joint 1)

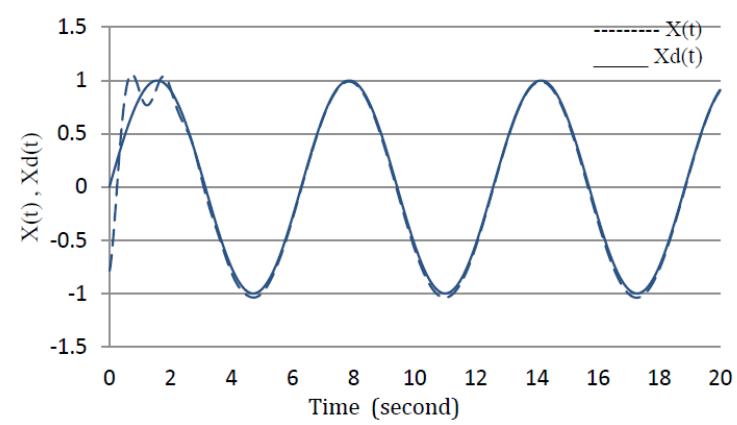

Fig. 7. Tracking response of $P D$ sliding mode control based on Multi objective Genetic Algorithm (joint 2)

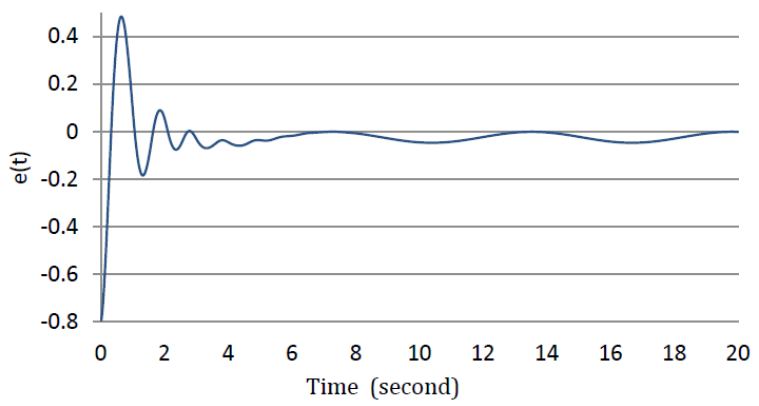

Fig. 8. Tracking error of $P D$ sliding mode control based on Multi objective Genetic Algorithm (joint 1)

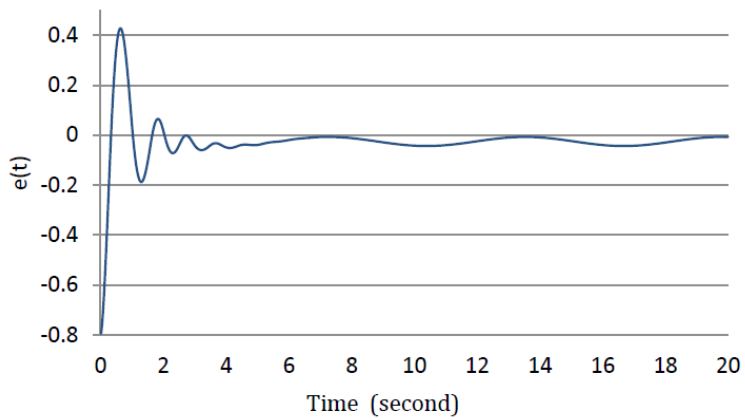

Fig. 9. Tracking error of $P D$ sliding mode control based on Multi objective Genetic Algorithm (joint 2)

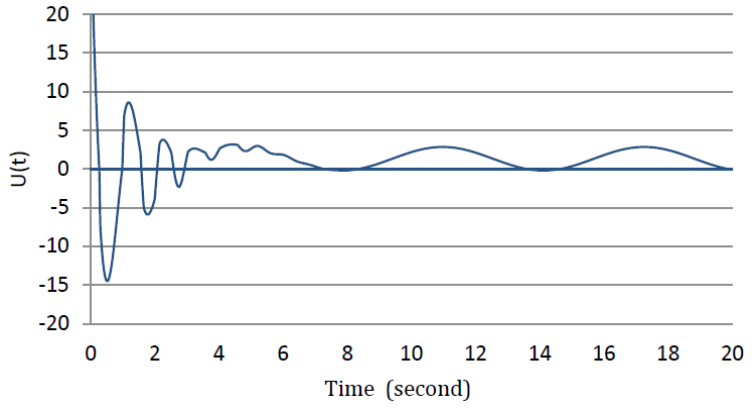

Fig. 10. Control input of $P D$ sliding mode control based on Multi objective Genetic Algorithm (joint 1)

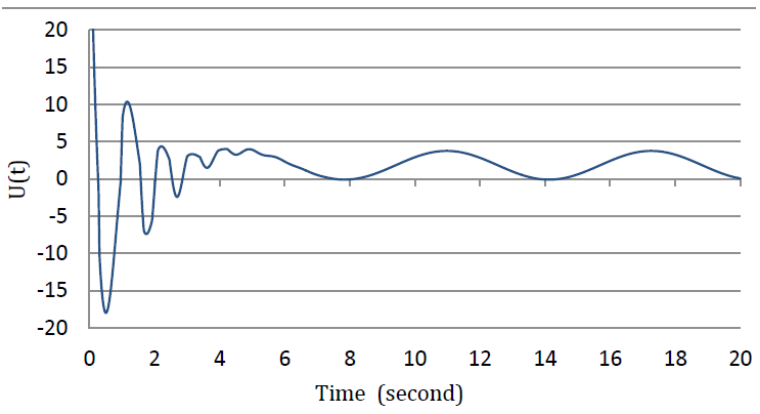

Fig. 11. Control input of $P D$ sliding mode control based on Multi objective Genetic Algorithm (joint 2)

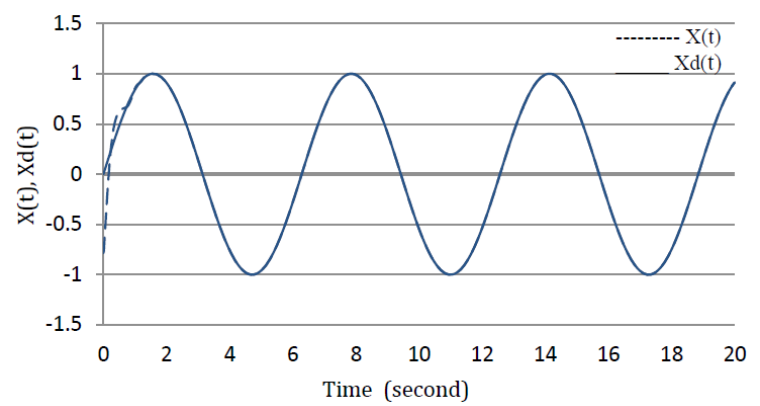

Fig. 12. Tracking response of $P D^{\alpha}$ sliding mode control based on Multi objective Genetic (joint 1)

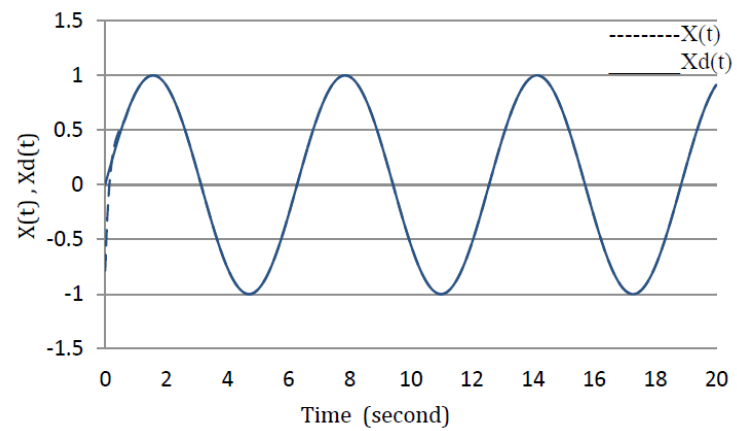

Fig. 13. Tracking response of $P D^{\alpha}$ sliding mode control based on Multi objective Genetic (joint 2) 


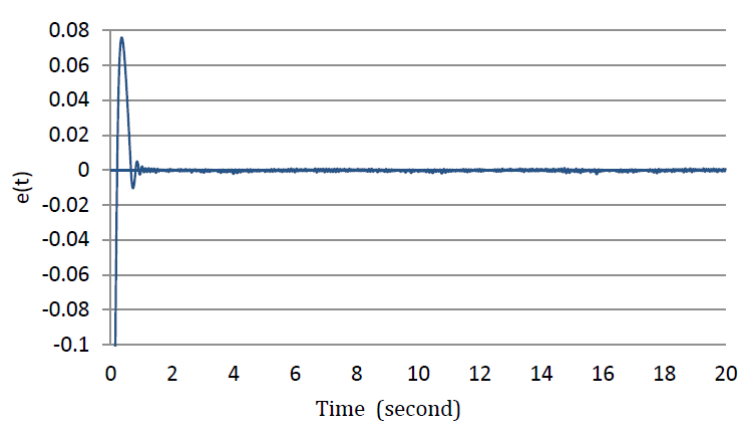

Fig. 14. Tracking error of $P D^{\alpha}$ sliding mode control based on Multi objective Genetic (joint 1)

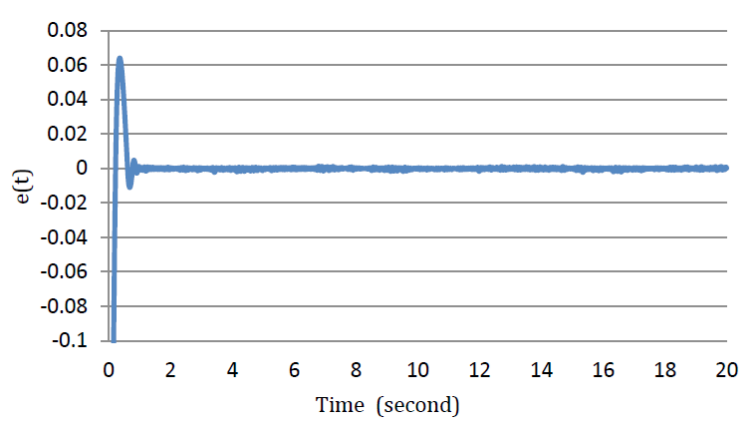

Fig. 15. Tracking error of $P D^{\alpha}$ sliding mode control based on Multi objective Genetic Algorithm (joint 2)

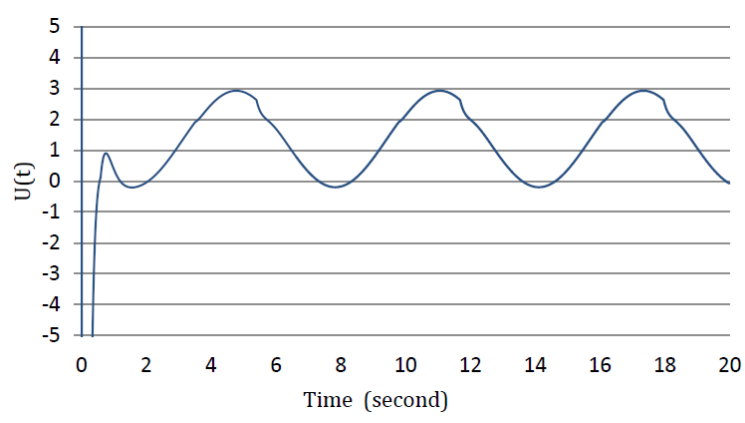

Fig. 16. Tracking response of $P D^{\alpha}$ sliding mode control based on Multi objective Genetic Algorithm (joint 1)

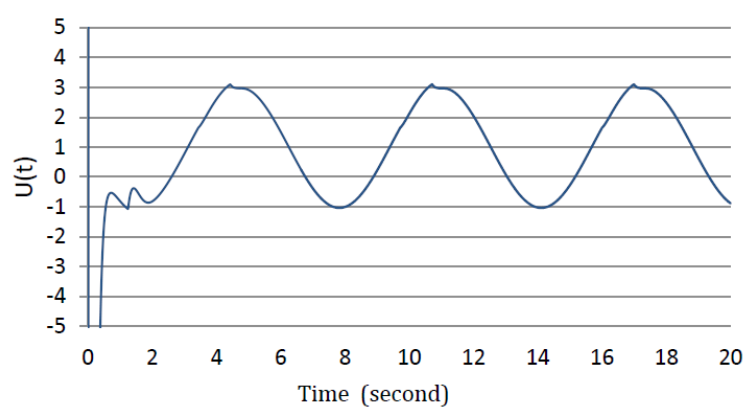

Fig. 17. Control input of $P D^{\alpha}$ sliding mode control based on Multi objective Genetic Algorithm (joint 2)
Moreover, the absolute total error of employing the $P D^{\alpha} \mathrm{SMC}$ is optimized in comparison with employing the PD SMC in Fig. 8, 9, 14 and 15.

From Table 2 can be seen that the reaching times $(1,2)$ and the absolute total errors $(E 1, E)$ are much less than $P D \mathrm{SMC}$ by employing the $P D^{\alpha}$ SMC.

Finally simulations results guarantee the genuineness of the suggested controller to boost the tracking performance of a nonlinear system and prove the robustness and efficiency of the $P D^{\alpha}$ SMC against model parameter uncertainty. And also chattering phenomena has been considered in Fig. 4 with regard using (17) when the state hit the sliding surface. Then by employing saturation function the chattering reduced significantly (Fig 10 and 11).

\section{CONCLUSION}

Pareto optimal design of fractional SMC developed for nonlinear system. The fractional order SMC based multi-objective GA was used to enable the system output tracks the desired reference trajectory and stabilize the system with tracking error. Proposed optimized controller offered superior properties such as faster finitetime convergence, higher control precision with very low control efforts and stability conditions guaranteed in control. By optimizing the controller, the satisfactory solution is selected in Pareto optimum solution set according to the system requirement. The results demonstrated that the optimized fractional SMC error was reduced significantly and tracking the desired value was conducted with higher accuracy. Finally, some numerical simulations are provided to confirm the validity of the proposed approach in various systems.

\section{REFERENCES}

1. Aghababa M. 2015. A fractional sliding mode for finite-time control scheme with application to stabilization of electrostatic and electromechanical transducers, Applied Mathematical Modelling, 39, 6103-6113.

2. Baleanu D., Güvenç Z.B. 2010. New trends in nanotechnology and fractional calculus applications. Dordrecht [etc.]. Springer.

3. Bisheban M., Mahmoodabadi M.J. 2013. Pareto optimal design of decoupled sliding mode control based on a new multi-objective particle swarm 
optimization algorithm. Amirkabir International Journal of Science \& Research, 45, 31-40.

4. Das S. 2007. Functional Fractional calculus for system identification and controls. Springer Publishing Company, Incorporated.

5. Deb K. 2001. Multi-objective optimization using evolutionary algorithms. John Wiley \& Sons.

6. Das S., Pan I. 2014. On the mixed H2/Hळ loopshaping tradeoffs in fractional-order control of the AVR system. IEEE Transactions on Industrial Informatics, 10, 1982-1991.

7. Deb K., Pratap A., Agarwal S., Meyarivan T. 2002. A fast and elitist multiobjective genetic algorithm: NSGA-II. Evolutionary Computation, IEEE Transactions on, 6(2), 182-197.

8. Emel'yanov S.V. 2007. Theory of variable-structure control systems: Inception and initial development. Computational Mathematics and Modeling, 18(4), 321-331.

9. Fonseca C.M., Fleming P.J. 1993. Genetic Algorithms for multiobjective optimization: formulationdiscussion and generalization. Paper presented at the ICGA.

10. Hamamci S.E. 2007. Stabilization using fractionalorder PI and PID controllers. Nonlinear Dynamics, 51(1), 329-343. DOI: 10.1007/s11071-007-9214-5.

11. Mahmoodabadi M.J., Bagheri A., Nariman-Zadeh N., Jamali A., Abedzadeh Maafi R. 2012. Pareto design of decoupled sliding-mode controllers for nonlinear systems based on a multiobjective genetic algorithm. Journal of Applied Mathematics.

12. Pan I., Das S., Das S. 2015. Multi-objective active control policy design for commensurate and incommensurate fractional order chaotic financial systems. Applied Mathematical Modelling, 39, 500-514.

13. Pan I., Das S. 2015. Designed a fractional-order PID controller for load-frequency control of two interconnected power systems.

14. Panigrahi B.K., Suganthan P.N., Das S., Dash, S.S. 2013. Swarm, Evolutionary, and Memetic Computing 4th International Conference, SEMCCO 2013, Chennai, India, December 19-21, 2013, Pro- ceedings, Part II. Retrieved from http://dx.doi. org/10.1007/978-3-319-03756-1.

15. Pashaki P.V., Pouya M. 2017. Investigation of high-speed cryogenic machining based on finite element approach. Latin American Journal of Solids and Structures. 14(4), 2017, 629-642.

16. Pashaki P.V., Pouya M. 2016. Volumetric error compensation in five-axis $\mathrm{CNC}$ machining center through kinematics modeling of geometric error. Advances in Science and Technology Research Journal, 10(30), 2016, 207-217. DOI: $10.12913 / 22998624 / 62921$.

17. Pashaki P.V., Pouya M., Maleki V.A. 2017. Highspeed cryogenic machining of the carbon nanotube reinforced nanocomposites: Finite element analysis and simulation. Proceedings of the Institution of Mechanical Engineers, Part C: Journal of Mechanical Engineering Science. Retrieved from https:// doi.org/10.1177/0954406217714012.

18. Perruquetti W. 2002. Sliding mode control in engineering. Marcel Dekker, Inc.

19. Podlubny I. 1999. Fractional differential equations an introduction to fractional derivatives, fractional differential equations, to methods of their solution and some of their applications. Retrieved from http://site.ebrary.com/id/10224992.

20. Sbalzarini I.F., Müller S., Koumoutsakos P. 2000. Multiobjective optimization using evolutionary algorithms. Paper presented at the Proceedings of the summer Program.

21. Valério D., da Costa J.S. 2006. Tuning of fractional PID controllers with Ziegler-Nichols-type rules. Signal Processing, 86(10), 2006, 2771-2784.

22. Zhong G., Deng H., Li J. 2015. Chattering-free variable structure controller design via fractional calculus approach and its application. Nonlinear Dynamics, 81, 679-694.

23. Zhong F., Li H., Zhong S. 2016. State estimation based on fractional order sliding mode observer method for a class of uncertain fractional-order nonlinear systems. Signal Processing, 127, 168-184.

24. Zinober A.S.I. 1989. Deterministic control of uncertain systems. London: Peregrinus. 